\title{
MiR-200b is upregulated in plasma-derived exosomes and functions as an oncogene by promoting macrophage M2 polarization in ovarian cancer
}

Jun Xiong, Xiaoju He, Yuanyuan Xu, Wei Zhang ${ }^{*}$ and Fen Fu ${ }^{*}$

\begin{abstract}
Background: Ovarian cancer is the seventh most common cancer in women and the second most reason of gynecologic cancer-related death. Growing evidence showed that exosomal miRNA plays a crucial role in the progression of ovarian cancer.

Methods: Exosomes were identified using nanoparticle tracking analysis, transmission electron microscopy and marker proteins detection. The levels of mRNA and proteins were ensured by qRT-PCR and western blot, respectively. Immunofluorescence, flow cytometry and ELISA assay were carried out to analyze macrophages polarization. CCK-8 and Transwell assay were used to measure the cell viability and invasion of ovarian cancer cells. The interaction of miR-200b and Kruppel like factor 6 (KLF6) was ensured by using luciferase reporter assay.

Results: Here, we obtained plasma-derived exosomes successfully, and proved that miR-200b was increased in the exosomes of ovarian cancer patients. Subsequently, our data showed that increasing of miR-200b could promote macrophage M2 polarization, but inhibit M1 polarization. miR-200b-overexpressed macrophages-conditioned medium notably enhanced the cell viability and invasion of ovarian cancer cells. Moreover, increasing of miR-200b inhibited KLF6 expression, while decreasing of miR-200b promoted KLF6 expression. Overexpression of KLF6 recused miR-200binduced macrophage polarization toward M2, and the inhibitory effect of miR-200b on M1 polarization.

Conclusions: Overall, our results demonstrated that miR-200b was highly expressed in the plasma-derived exosome of ovarian cancer patients, and promoted the proliferation and invasion of ovarian cancer cells through inducing macrophage M2 polarization by suppressing KLF6 expression. Our results suggested that miR-200b might be a novel target for ovarian cancer treatment.
\end{abstract}

Keywords: Ovarian cancer, MiR-200b, Macrophage polarization, Kruppel like factor 6

\footnotetext{
* Correspondence: zhang_wei723@sohu.com; fufen_nc@163.com

Department of Obstetrics and Gynecology, The Second Affiliated Hospital of Nanchang University, No.1 Minde Road, Donghu District, 330006 Nanchang, Jiangxi, China
}

C C The Author(s). 2021 Open Access This article is licensed under a Creative Commons Attribution 4.0 International License, which permits use, sharing, adaptation, distribution and reproduction in any medium or format, as long as you give appropriate credit to the original author(s) and the source, provide a link to the Creative Commons licence, and indicate if changes were made. The images or other third party material in this article are included in the article's Creative Commons licence, unless indicated otherwise in a credit line to the material. If material is not included in the article's Creative Commons licence and your intended use is not permitted by statutory regulation or exceeds the permitted use, you will need to obtain permission directly from the copyright holder. To view a copy of this licence, visit http://creativecommons.org/licenses/by/4.0/ The Creative Commons Public Domain Dedication waiver (http://creativecommons.org/publicdomain/zero/1.0/) applies to the data made available in this article, unless otherwise stated in a credit line to the data. 


\section{Background}

Ovarian cancer is the seventh most common cancer in women, and it is the second most reason of gynecologic cancer-related death and the eighth most common reason of cancer-related death in women globally. The fiveyear survival rate of ovarian cancer patients is approximately $47 \%[1,2]$. Epithelial ovarian cancer (EOC) is the major type of ovarian cancer, accounting for $85-90 \%$ of total case. According to the origin, pathogenesis, risk factors, prognosis and molecular alterations of ovarian cancer, it can be divided into five subtypes [3, 4]. Generally, early in ovarian cancer patients is not easy to be found, hence, the majority of patients with clinical advanced stage when they were diagnosed. For the patients with advanced ovarian cancer, surgical treatment and chemotherapy are the major therapeutic methods, while the resistance to chemotherapeutic drugs greatly declines the outcome of the patients $[5,6]$. Thereby, it is very necessary to find a new target for ovarian cancer treatment.

Exosomes are one of the type of vesicles with 30-150 $\mathrm{nm}$ of diameter, which can be released by almost all cell types such as tumor cancer cell and macrophage. Exosome are an important messenger among different cells [7]. In recent years, more and more studies have indicated that exosomes play a crucial role in the development of multiple diseases, including ovarian cancer [8]. It was reported that exosomes could transmit messages to target cells through carrying microRNA (miRNA), protein and other moleculars, thus to involve in many biological process like communication in intercellular, cell differentiation and immune modulation [9]. Growing evidence that miRNA, a class of endogenous non-coding RNA, can be acted as one of the index in tumor detection and a potential target for tumor treatment [10]. For instance, Zhang et al. demonstrated that miR-337-3p is decreased in EOC tissues. MiR-337-3p promoted EOC cell cycle arrest in G0/G1 phase and apoptosis and inhibited cell proliferation, suggesting miR-337-3p is a potential therapeutic target of EOC [11]. Recently, Pan et al. found that numerous miRNAs are abnormally expressed in the plasma-derived exosome of the EOC patients, such as miR-200b [12]. However, whether exosomal miR-200b participates in regulating the development of ovarian cancer remains unclear.

Some studies indicated that exosomal miRNAs could affect the progression of cancer through mediating the interaction of cancer cells and immune cells [13]. Macrophages are an important class of immune cells in tumor microenvironment. In accordance with different extracellular environment, macrophages can differentiate into $M$ macrophages and M2 macrophages [14]. It was proved that M1 macrophages suppress cancer progression through secreting pro-inflammatory cytokines, whereas
M2 macrophages promote cancer progression via secreting anti-inflammatory cytokines $[15,16]$. A recent study indicated that miR-21 is increased in ovarian cancer tissues, and it enhances the chemoresistance of ovarian cancer cells through facilitating macrophage M2 polarization, indicating the crucial role of miRNA in the interaction of macrophage and ovarian cancer cells [17]. In this present study, we also proved that miR-200b was highly expressed in the exosomes from the plasma of EOC patients, and indicated that miR-200b promoted ovarian cancer cell proliferation and invasion via promoting macrophages M2 polarization and inhibiting M1 polarization by repressing Kruppel like factor 6 (KLF6) expression. Our data revealed firstly that the regulatory mechanism of miR-200b in ovarian cancer proliferation, and suggested that miR-200b might a potential target for ovarian cancer treatment.

\section{Materials and methods}

\section{Exosome isolation and identification}

All participants provided the written informed consent. This study was approved by the Second Affiliated Hospital of Nanchang University. For exosome isolation, peripheral blood samples were taken from three healthy women, and six EOC patients who accepted with therapy in our hospital. Then, ExoQuick ${ }^{\mathrm{Tm}}$ Exosome Precipitation Solution (System Biosciences, Mountain View, Calif) was utilized to extract exosomes from the plasma in accordance with the manufacture's introduction. Transmission electron microscopy (TEM) was utilized to analyze the morphology of exosomes, and the size and concentration of exosomes was ensured by nanoparticle tracking analysis (NTA) on a NanoSight NS300 (Malvern Instrument Ltd.). Besides, the expression of the markers of exosomes, including lysosome-associated membrane proteins 1 (LAMP-1) and tumour susceptibility gene 101 (TSG101), were measured by using western blot.

\section{Cell culture and treatment}

Human monocytic cell line THP-1 was purchased from the American Type Culture Collection (ATCC, USA). THP-1 cells were cultured in RPMI-1640 medium containing $10 \%$ fetal bovine serum (FBS, Gibco, New York, USA), $1 \%$ streptomycin and penicillin (Gibco), and 0.05 $\mathrm{nM} \beta$-mercaptoethanol (Gibco). $50 \mathrm{ng} / \mathrm{ml}$ of phorbol12-myristate 13-acetate (PMA, Sigma-Aldrich, Missouri, USA) was used to induce the differentiation of THP-1 cells toward macrophages. After incubation with PMA for $48 \mathrm{~h}$, the THP-1 cells were collected, named as M0 macrophage, and then were used in subsequent studies. In our present study. To explore the effect of miR-200b on macrophage polarization, we obtained miR-200b mimic and mimic negative control (mimic NC), and miR-200b inhibitor and inhibitor negative control (inhibitor NC) from RiboBio (Guangzhou, China). Besides, 
we obtained the lentiviruses packaged with KLF6 overexpression vector (LV-KLF6) and the lentiviruses packaged with empty vector (LV-NC) from BrainVTA (Wuhan, China). Here, $50 \mathrm{nM}$ of miR-200b mimic, $50 \mathrm{nM}$ of mimic NC, $100 \mathrm{nM}$ of miR-200b inhibitor and $100 \mathrm{nM}$ of inhibitor $\mathrm{NC}$ were transfected into M0 macrophages using the transfection reagent Lipofectamine 2000 (Invitrogen, Carlsbad, USA) according to the protocol. After $24 \mathrm{~h}$ of cell transfection, qRT-PCR, western blot and other experiments were carried out.

Human ovarian carcinoma cell line OVCAR-3 also was obtained from ATCC, and the cells were maintained in RPMI-1640 medium supplemented with $10 \%$ FBS and $1 \%$ streptomycin and penicillin. After $24 \mathrm{~h}$ of miR200b mimic and mimic NC transfection, the conditioned cell culture medium of M0 macrophages, mimic NCtransfected M0 macrophages and miR-200b mimictransfected M0 macrophages were collected, and then were used to culture OVCAR-3 cells. PBS-treated OVCAR-3 cells acted as control group. At $24 \mathrm{~h}$ later of incubation, cell proliferation and cell invasion were measured. In our study, all cells were cultured at $37^{\circ} \mathrm{C}$ in an incubator with $5 \% \mathrm{CO}_{2}$.

\section{Western blot}

The expression of LAMP-1 and TSG101 in exosomes, and the expression of KLF6 in M0 macrophages were measured by using western blot. RIPA lysis buffer was used to isolate total protein. Equal quality $25 \mu \mathrm{g}$ of cellular protein from each group were separated on the $12 \%$ SDS-PAGE gel, and then were transferred to a PVDF membrane. After that, membranes were blocked with $5 \%$ non-fat mile for $1 \mathrm{~h}$ at room temperature and subsequent primary antibodies overnight at $4{ }^{\circ} \mathrm{C}$. The primary antibodies were as follows: anti-LAMP-1 antibody (1: 1000, Cell Signaling Technology, Boston, MA, USA), anti-TSG101 antibody (1:1000, Cell Signaling Technology) and anti-KLF6 antibody (1:1000, Cell Signaling Technology). After incubation with primary antibodies, the membranes were maintained with secondary antibodies (1:3000, Abcam) for $1 \mathrm{~h}$ at room temperature. At last, the membranes were maintained with the enhanced chemiluminescence reagent (Merck Millipore, Missouri, USA) to detect the protein bands. The relative expression level of KLF6 was analyzed utilizing Image J software (National Institutes of Health, Maryland, USA). Here, $\beta$-actin served as the internal reference of KLF6.

\section{QRT-PCR}

Total RNA was extracted from M0 macrophages using TRIzol reagent (Invitrogen) in accordance with the manufacture's introduction. Then, the concentration of total RNA was ensured utilizing the NanoDrop 2000, and $1 \mu \mathrm{g}$ of total RNA was added into the reaction system of reverse transcription. PrimeScript RT reagent kit (TaKaRa) was used to synthesis complementary DNA according to the manufacture's protocol. After that, realtime PCR was implemented on ABI 7300HT instrument (Applied Biosystems) using the SYBRR Green Supermix kit $(\mathrm{TaKaRa})$ in accordance with the protocol. The relative expression levels of KLF6, iNOS and Arg-1 mRNAs were normalized to GAPDH, and U6 served as the internal reference of miR-200b.

\section{Immunofluorescence analysis}

M0 macrophages were planted at the density of $1 \times 10^{5}$ cells per well into a 24-well plate and subsequent transfected with miR-200b mimic and mimic NC for $24 \mathrm{~h}$. Next, the cells were fixed with $4 \%$ paraformaldehyde (Solarbio, Beijing, China) for $30 \mathrm{~min}$ at $4{ }^{\circ} \mathrm{C}$ and subsequent incubated with $0.5 \%$ Triton X-100 for 20 min at room temperature. After that, the cells were incubated with $2 \%$ BSA for 30 min followed by primary antibodies, including anti-CD86 antibody (1:200, Abcam, Cambridge, MA, USA) and anti-CD206 antibody (1:200, Abcam) overnight at $4{ }^{\circ} \mathrm{C}$. Next day, the cells were maintained with secondary antibodies, including Alexa Fluor-488-conjugated goat anti-rabbit IgG antibody (1:1000, Abcam) and Alexa Fluor674-conjuated goat anti-mouse IgG antibody (1:1000, Abcam), for $1 \mathrm{~h}$ at room temperature in the dark. Next, the cells were stained with DAPI reagent for $5 \mathrm{~min}$ in the dark. At last, the CD86-positive and CD206-positive cells were observed under a confocal microscope (Leica). The red and blue images were overlaid to create the merge images.

\section{Flow cytometry}

M0 macrophages were planted at the density of $1 \times 10^{5}$ cells per well into a 24-well plates. After $24 \mathrm{~h}$ of cell transfection and lentivirus infection, the cells were collected for flow cytometry. The cells were maintained with PE-labeled CD206 and FITC-labeled CD86 antibodies for $30 \mathrm{~min}$ at room temperature. Next, the stained cells were analyzed using a BD Accuri ${ }^{\text {Tim }}$ C6 cytometer (BD Biosciences).

\section{ELISA assay}

ELISA assay was carried out to measure the concentration of interleukin-1 $\beta$ (IL-1 $\beta$ ) and C-C motif chemokine ligand 17 (CCL17) in cell culture supernatants. ELISA assay was accomplished according to manufacturer's protocols of human IL-1 $\beta$ ELISA kit (R\&D systems, Minneapolis, USA) and human CCL17 ELISA kit (R\&D systems).

\section{CCK-8 assay}

Cancer cells were planted at the density of $6 \times 10^{3}$ cells per well into 96 -well plates. M0 macrophages-, mimic 
NC-transfected M0 macrophages- and miR-200b mimictransfected M0 macrophages-conditioned medium were used to culture cancer cells. At $24 \mathrm{~h}$ later of cell culture, the cells were maintained with $10 \mu \mathrm{l}$ of CCK solution (Solarbio) for another $2 \mathrm{~h}$ at $37{ }^{\circ} \mathrm{C}$. Then, the optical density of each well at $450 \mathrm{~nm}$ were measured using a microplate reader (Elx800; BioTek Inc., North Brunswick, NJ, USA).

\section{Transwell invasion assay}

The bottom of the upper chambers of 24-well transwell chambers (Corning Incorporated, Corning, NY, USA) were pre-coated with matrigel. $1 \times 10^{4}$ cancer cells were seeded into the upper chamber, and were cultured with serum-free RPMI-1640 medium. The 1:1 mixture of macrophages-conditioned medium and fresh RPMI-1640 medium containing $15 \%$ FBS was added into the lower chambers. At $24 \mathrm{~h}$ later of cell culture, the invaded cancer cells were fixed with $10 \%$ methanol and then stained with $0.1 \%$ crystal violet. At last, the stained cancer cells were observed using a microscope.

\section{Luciferase reporter assay}

The binding sites between miR-200b and KLF6 3'-UTR were predicted using starBase v2.0 database. The wildtype (wt) and mutant (mut) gene sequences of KLF6 3'UTR containing the binding sites with miR-200b were sub-cloned into p-GL3 vector. Then, KLF6 3'-UTR wt vector or KLF6 3'-UTR mut vector were co-transfected into HEK293T cells with miR-200b mimic or mimic NC. At $24 \mathrm{~h}$ later of transfection, the luciferase activity of cells were measured. In the luciferase reporter assay, we used two negative control (mimic NC and luciferase reporter lacking the KLF6 3'-UTR).

\section{Statistical analysis}

All data analysis were performed using SPSS 22.0 software, and expressed as mean \pm SD. The significant difference between two independent groups was analyzed using Student $t$-test, and the significant difference among multiple groups was analyzed using one-way ANOVA. The value of P lower than 0.05 as considered statistically significant.

\section{Results}

miR-200b was highly expressed in the plasma-derived exosome of EOC patients

To verify the expression levels of miR-200b in the plasma-derived exosomes of healthy women and EOC patients, we isolated the exosomes from the plasma of 3 cases of healthy women and six cases of EOC patients. The typical circle and saucer-like structures characteristic of exosomes were observed using a TEM (Fig. 1a), and the diameters of exosomes lower than $100 \mathrm{~nm}$ was identified by NTA (Fig. 1b). In addition, we found that the markers of exosomes, including LAMP-1 and TSG101, expressed in the plasma-derived vesicle (Fig. 1c). Importantly, our results showed that the level of miR-200b was higher in the plasma-derived exosomes of EOC patients than that in the plasma-derived exosomes of healthy women (Fig. 1d).

\section{miR-200b promoted macrophage M2 polarization, while inhibited M1 polarization}

To explore the effect of miR-200b on the polarization of macrophage, we designed the miR-200b mimic and mimic NC. Our data showed that the expression of miR200b was significantly promoted by miR-200b mimic treatment in M0 macrophages (Fig. 2a). Subsequently, we detected the polarization of macrophages toward M2 and M1. The results of immunofluorescence indicated that the number of CD86-positive cells (M1-polarized macrophages) was notably reduced, whereas the number of CD206-positive cells (M2-polarized macrophages) was increased in M0 macrophages by miR-200b mimic treatment (Fig. 2b). Flow cytometry results also demonstrated that the percentage of CD86-positive cells was downregulated, but the percentage of CD206-positive cells was significantly upregulated by miR-200b mimic treatment (Fig. 2c and d). Moreover, we further found that the level of iNOS mRNA was lower, while the level of Arg-1 mRNA was higher in the M0 macrophages transfected with miR200b mimic compared to the M0 macrophages transfected with mimic $\mathrm{NC}$ (Fig. 2e, f). The concentration of M1 macrophages-related cytokine (IL-1 $)$ was declined, and the concentration of M2 macrophages-related cytokine (CCL17) was increased in the cell supernatants of miR200b mimic-treated M0 macrophages (Fig. 2g, h). In summary, miR-200b promoted macrophage polarization toward M2, and inhibited macrophage polarization toward M1.

miR-200b facilitated EOC cells proliferation and invasion Furthermore, at $24 \mathrm{~h}$ after miR-200b mimic and mimic $\mathrm{NC}$ transfection, we collected the culture medium of M0 macrophages, mimic NC-treated M0 macrophages and miR-200b-transfected M0 macrophages. EOC cells were incubated with above cell culture medium for 24 h. CCK-8 assay demonstrated that M0 macrophages-conditioned medium suppressed the cell viability of EOC cells, while miR-200b increasing notably facilitated the cell viability of EOC cells (Fig. 3a). In addition, M0 macrophages-conditioned medium inhibited the invasion of EOC cells, but miR-200b-overexpressed M0 macrophages-conditioned medium markedly exacerbated the invasion of EOC cells (Fig. 3b). Overall, miR$200 \mathrm{~b}$ promoted EOC cells proliferation and invasion through inducing macrophage M2 polarization. 

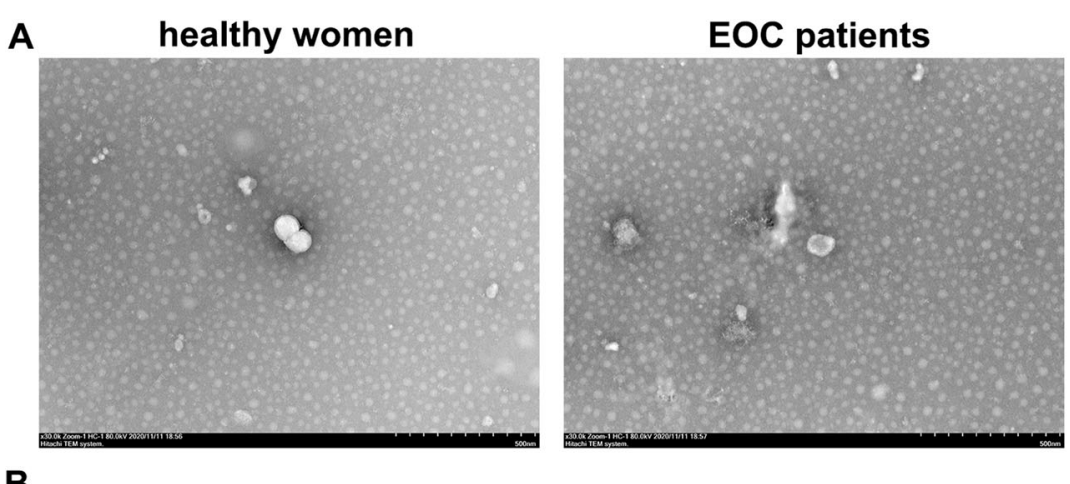

B
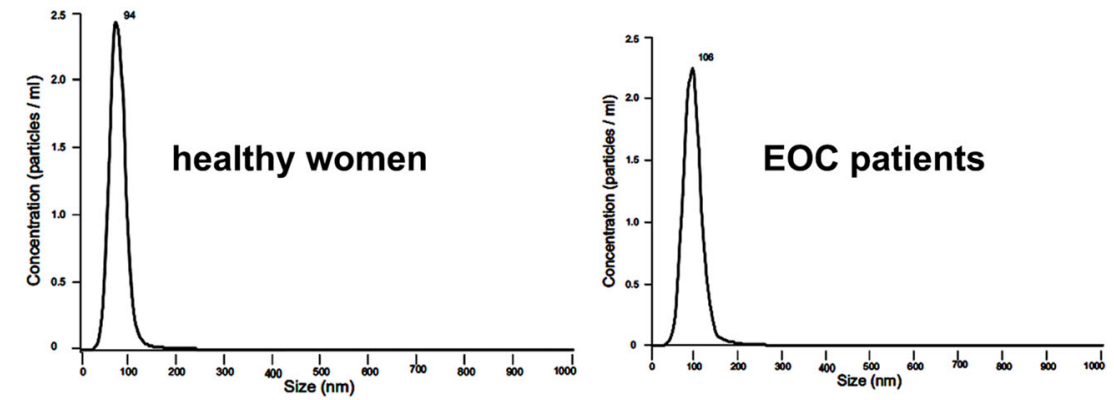

C healthy women

EOC patients
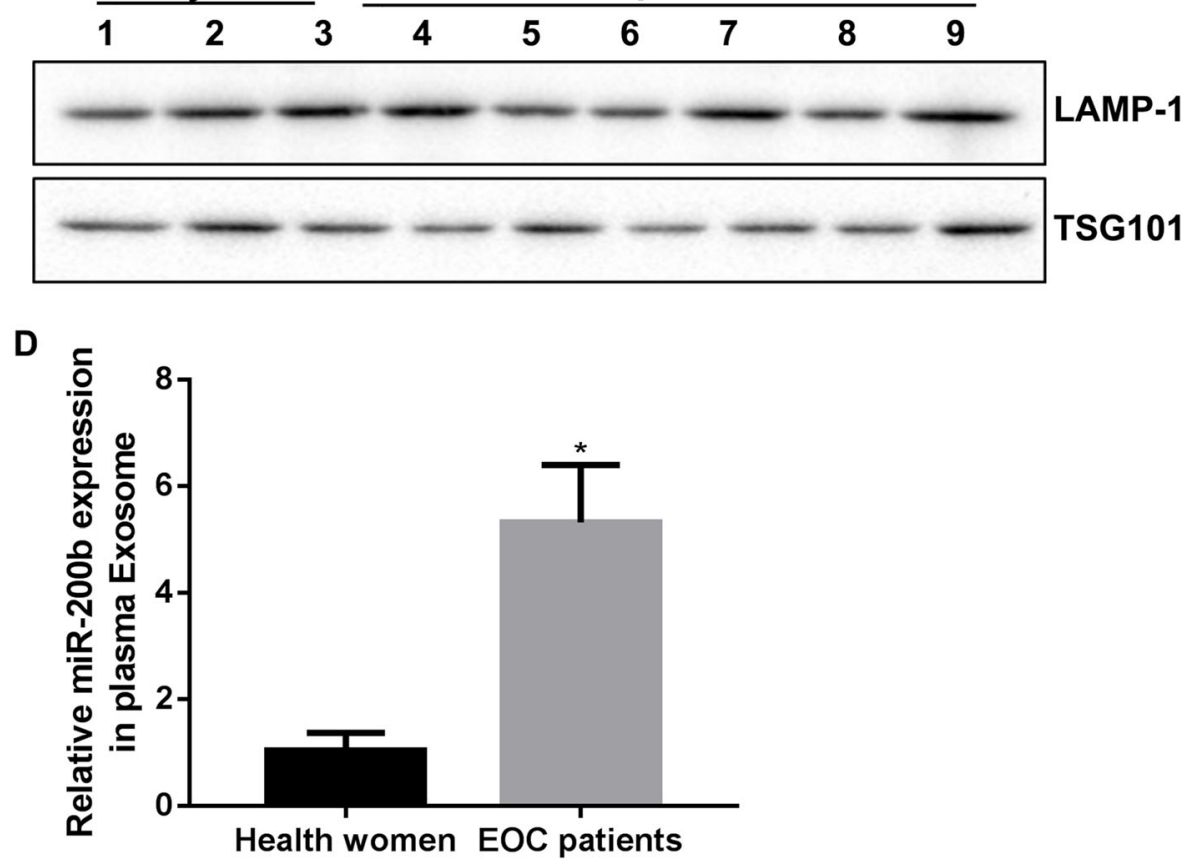

Fig. 1 The expression of miR-200b in the plasma-derived exosomes. Exosomes were isolated from the plasma of healthy women and EOC patients. a TEM was used to observe the morphology of exosomes. b NTA was used to analyze the size and concentration of exosomes. $\mathbf{c}$ The markers of exosomes, including LAMP-1 and TSG101, were detected by western blot. $\mathbf{d}$ qRT-PCR was carried out to measure the level of miR-

miR-200b regulated macrophage polarization through inhibiting KLF6

We predicated the binding sites of human miR-200b and KLF6 3'-UTR using starBase website (Fig. 4a), and proved the combination of miR-200b and KLF6 3'-UTR
(Fig. 4b). Moreover, our results showed that increasing of miR-200b significantly declined the levels of KLF6 mRNA and protein, while decreasing of miR-200b markedly upregulated the levels of KLF6 mRNA and protein in M0 macrophages (Fig. 4c-e). Then, we explore 

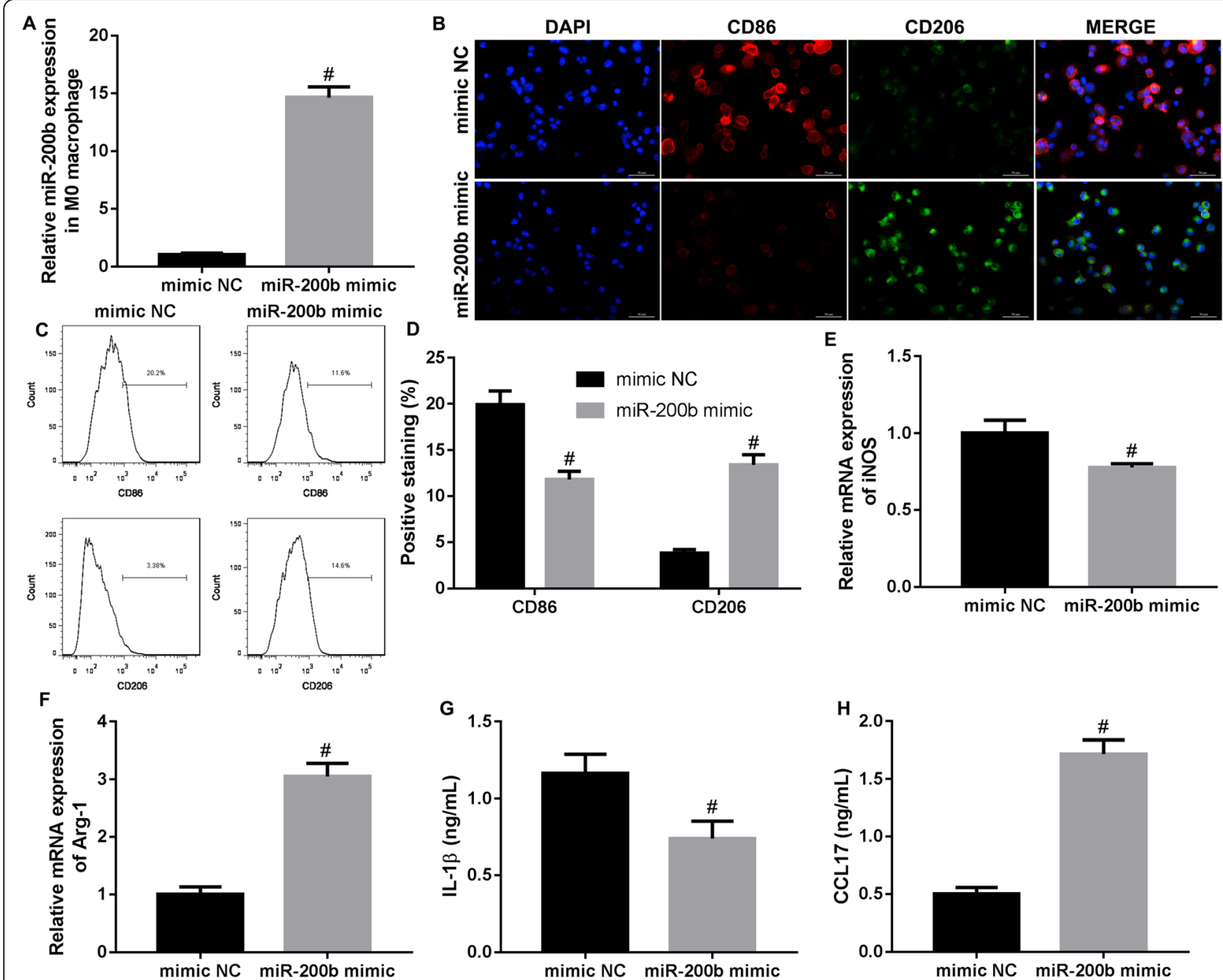

Fig. 2 The effect of miR-200b on macrophages polarization. miR-200b mimic and mimic NC were transfected into M0 macrophages. At 24 hours after transfection, a qRT-PCR was performed to examine the expression of miR-200b in the cells. $\mathbf{b}$ and $\mathbf{c}$ Immunofluorescence and flow cytometry were utilized to detect the number of CD86-positive cells and CD206-positive cells. $\mathbf{d}$ The percentages of CD86-positive and CD206positive macrophages were analyzed in accordance with the results of flow cytometry. e and $\mathbf{f}$ The levels of iNOS mRNA and Arg-1 mRNA were measured by qRT-PCR. $\mathbf{g}$ and $\mathbf{h}$ The concentrations of IL-1 $\beta$ and CCL17 in cell supernatant of macrophages were measured by ELISA assay. ${ }^{\#} P<$ 0.05 contrasted with mimic NC

whether miR-200b affects macrophage polarization through inhibiting KLF6. M0 macrophages were infected with the lentivirus expressing KLF6 following miR-200b mimic transfection. Our data revealed that the inhibition of miR-200b to macrophage M1 polarization, and the promotion of miR-200b to macrophage M2 polarization were rescued by KLF6 overexpression (Fig. 4f-h). The inhibition of miR-200b to M1 macrophages-related protein iNOS expression, and the promotion of miR200b to M2 macrophages-related protein Arg-1 expression were reversed by KLF6 overexpression (Fig. 4i, j). Furthermore, our resulted indicated that miR-200b reduced the production of M1 macrophages-related cytokine IL-1 $\beta$, and facilitated the production of M2 macrophages-related cytokine CCL17 in M0 macrophages, while which were recused following overexpression of KLF6 (Fig. 4k and l). In conclusion, miR200b promoted macrophage M2 polarization and inhibited M1 polarization through suppressing KLF6.

\section{Discussion}

Exosomes are extracellular lipid bilayer vesicles with a diameter of $30-100 \mathrm{~nm}$, released by almost all cells in physiological and pathological conditions. The exosome isolated from biological fluids including plasma may act as a liquid biopsy to assist the diagnosis of cancer [18]. LAMP-1, TSG101, CD86 and heat shock proteins 70 are the important characteristic molecules. Currently, TEM, NTA and western blot are the major methods for exosome identification $[19,20]$. Here, we determined using 


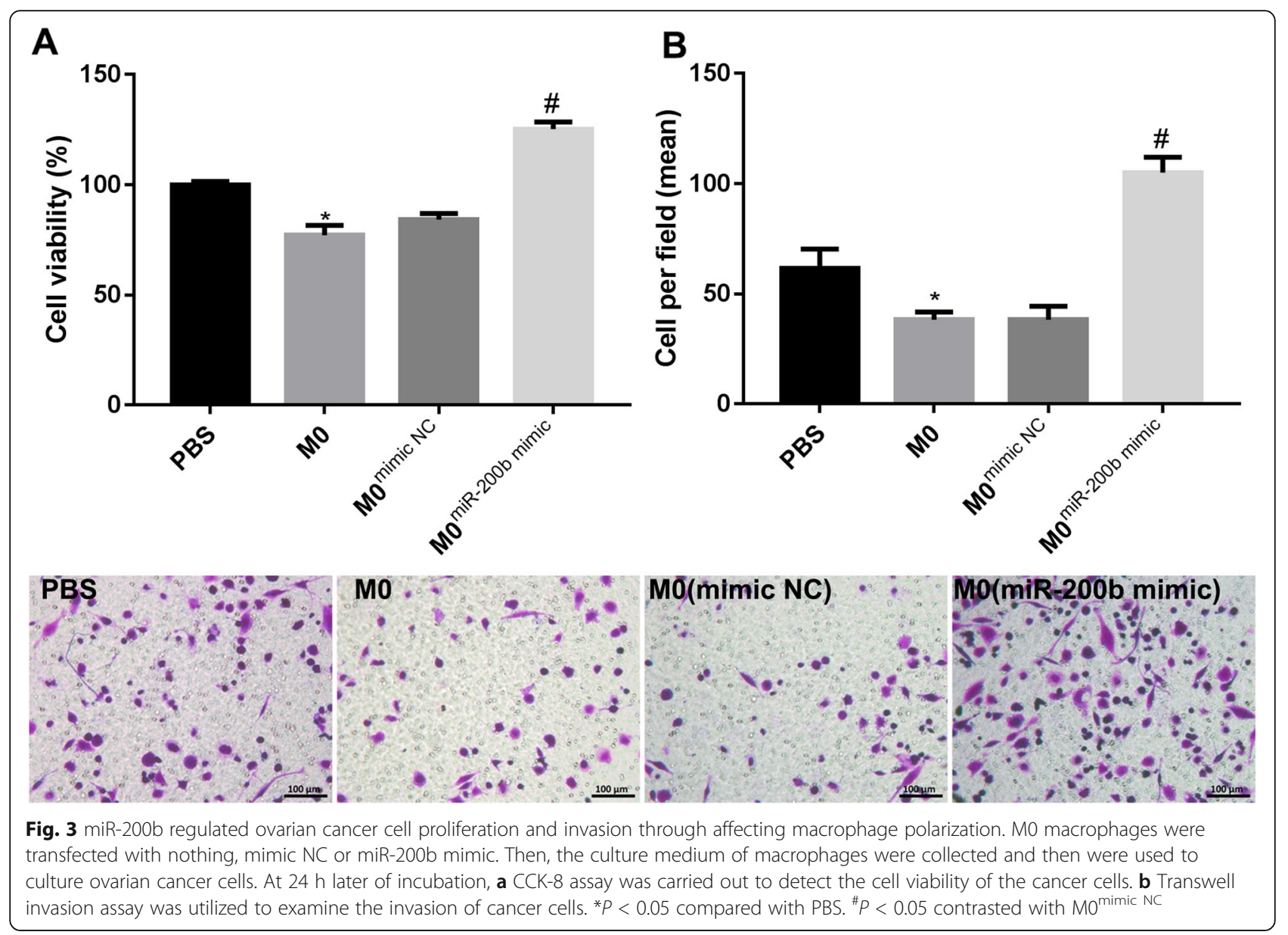

above methods that we obtained the plasma-derived exosomes successfully. It was known that exosomes play their biological function via carrying numerous molecules, such as protein, miRNA and circRNA, thus to involve in the development of many disorders [21]. For instance, ovarian cancer cells changed the morphology of human peritoneal mesothelial cells (HPMCs) to a mesenchymal phenotype via transferring exosomes to HPMCs. Subsequently, the HPMCs facilitated the invasion of ovarian cancer cells via submitting CD44-overexpressed exosomes to the cancer cells [22]. Furthermore, exosomal miR-99a-5p was highly expressed in the serum of EOC patients. EOC cells-derived exosomal miR-99a-5p could effectively facilitate the invasion of cancer cells through regulating HPMCs by promoting fibronectin and fibronectin, suggesting exosomal miRNA might be a potential target for ovarian cancer diagnose and treatment [23]. In our present study, we found that miR-200b was increased in the plasma-derived exosomes of EOC patients, and explored the action mechanism of miR- 200b regulating ovarian cancer cell proliferation and invasion.

Several previous studies have indicated that miR-200b is high expressed in the serum of EOC patients compared with healthy women, and the serum miR-200b level is associated with the grade of EOC $[24,25]$. However, a recent research proved that miR-200b is lower expressed in ovarian cancer cells when compared to the human ovarian fibroblasts, and it suppresses the proliferation of cancer cells through targeting PI3K/AKT signaling pathway [26]. The role and action mechanism of miR-200b in ovarian cancer still not clear. M1 macrophages can be induced by interferon- $\gamma$, and highly secrete IL-1 $\beta, \quad$ IL-12 and other pro-inflammatory cytokines. M2 macrophages can be induced by IL- 4 , IL-13 and IL-10, and highly express CCL17, IL-10 and other anti-inflammatory cytokines [27]. The polarization of macrophages toward M1 or M2 is closely related to the progression of cancer. For instance, miR-130a was increased in M1 macrophages, and it was proved to impede the progression of non- 


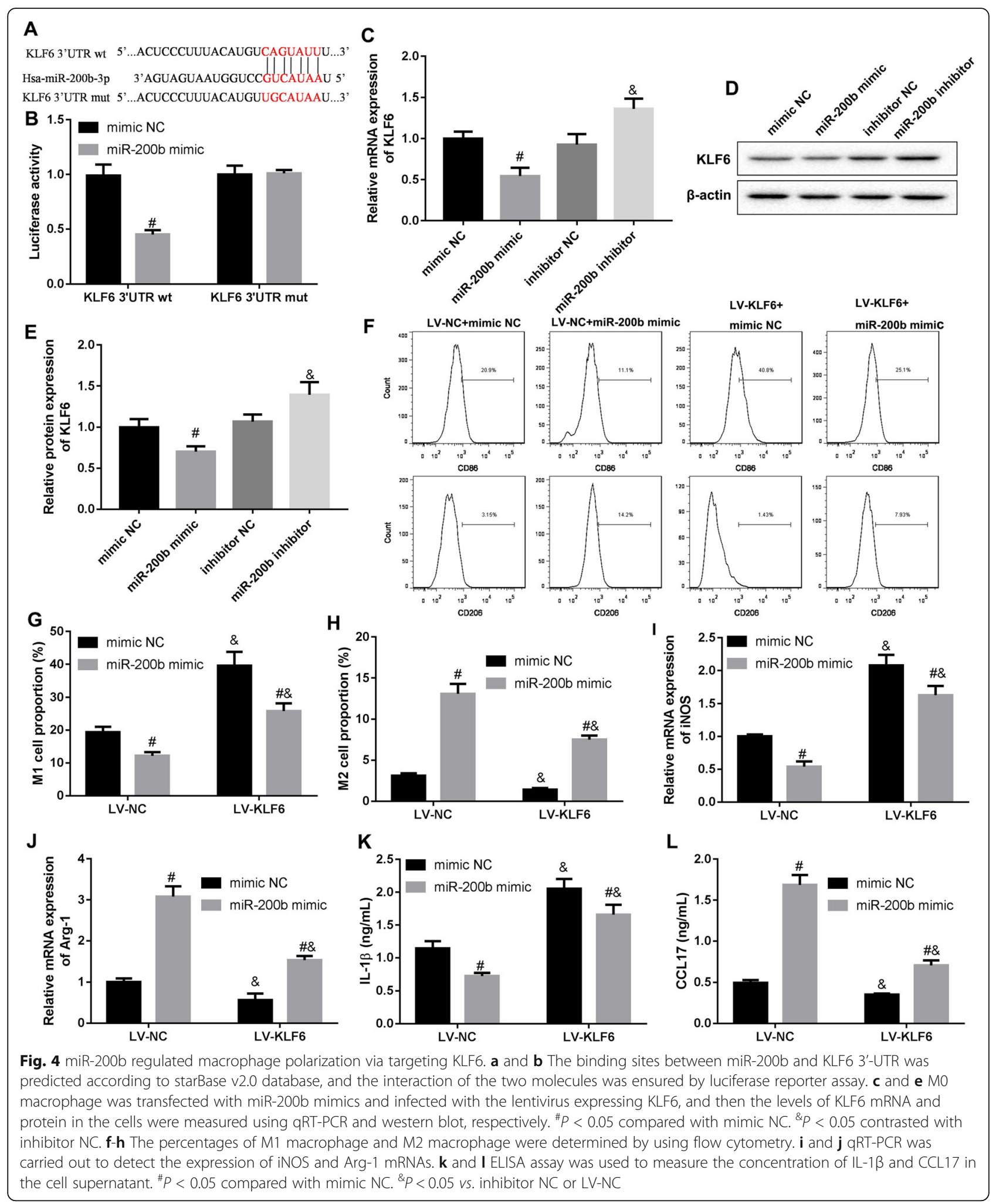

small cell lung cancer through promoting the polarization of macrophages toward M1 and inhibiting M2 polarization [28]. M2 macrophages contribute the proliferation of ovarian cancer cells [29]. Besides, it was revealed that inhibiting the differentiation of macrophages into M2 macrophages could effectively suppress the progression of ovarian cancer [30]. In this present study, our data showed that miR-200b 
inhibited macrophage M1 polarization and facilitated M2 macrophage, thus to promote the ovarian cancer cell proliferation and invasion.

KLF6 is a member of KLFs family, which are the highly conserved zinc-finger proteins. Growing evidence proved that KLF6 acts as a tumor suppressor molecular in multiple malignant cancers [31]. KLF6 was proved to impede macrophages M2 polarization and M2 macrophages-mediated tumor metastasis [32]. In ovarian cancer, some molecules could accelerate the development of the disease through binding with the 3'-UTR region of KLF6 and then suppressing the expression of KLF6, for instance miR-630 [33]. Here, we found that miR-200b promoted macrophages M2 polarization through suppressing KLF6 expression.

\section{Conclusions}

Overall, our data indicated that miR-200b was increased in the plasma-derived exosomes of EOC patients, and it boosted ovarian cancer cell proliferation and invasion via inducing macrophages M2 polarization by suppressing KLF6 expression. Our results might provide a novel target for ovarian cancer treatment.

\section{Acknowledgements}

Not applicable.

\section{Authors' contributions}

JX designed the study; JX, XH, YX, WZ participated in the experiments; WZ and FF analyzed the data; FF drafted the paper. All authors approved the paper.

\section{Funding}

This work was supported by the Science and Technology Research Project of Education Department of Jiangxi Province (190141).

\section{Availability of data and materials}

The datasets used and/or analysed during the current study are available from the corresponding author on reasonable request.

\section{Declarations}

\section{Ethics approval and consent to participate}

This study was approved by the Second Affiliated Hospital of Nanchang University. All participants provided the written informed consent.

\section{Consent for publication}

Not applicable.

\section{Competing interests}

The authors declare that they have no competing interests.

Received: 8 April 2021 Accepted: 12 May 2021

Published online: 02 June 2021

\section{References}

1. Moufarrij S, Dandapani M, Arthofer E, Gomez S, Srivastava A, Lopez-Acevedo M, Villagra A, Chiappinelli KB. Epigenetic therapy for ovarian cancer: promise and progress. Clinical epigenetics. 2019;11(1):7.

2. Lheureux S, Braunstein M. Epithelial ovarian cancer: Evolution of management in the era of precision medicine. CA Cancer J Clin. 2019; 69(4):280-304.

3. Wang W, Liu JR, Zou W. Immunotherapy in ovarian cancer. Surg Oncol Clin N Am. 2019;28(3):447-64.
4. Langdon SP, Gourley C, Gabra H, Stanley B. Endocrine therapy in epithelial ovarian cancer. Expert Rev Anticancer Ther. 2017;17(2):109-17.

5. Eisenhauer EA. Real-world evidence in the treatment of ovarian cancer. Ann Oncol. 2017:28(suppl_8):viii61-5.

6. Kossaï M, Leary A, Scoazec JY, Genestie C. Ovarian cancer: a heterogeneous disease. Pathobiol J ImmunoPathol Mol Cell Biol. 2018;85(1-2):41-9.

7. Koritzinsky EH, Street JM, Star RA, Yuen PS. Quantification of exosomes. J Cell Physiol. 2017;232(7):1587-90.

8. Feng W, Dean DC, Hornicek FJ, Shi H, Duan Z. Exosomes promote pre-metastatic niche formation in ovarian cancer. Mol Cancer. 2019; 18(1):124.

9. Klingeborn M, Dismuke WM, Bowes Rickman C, Stamer WD. Roles of exosomes in the normal and diseased eye. Prog Retin Eye Res. 2017;59:158-77.

10. Ghafouri-Fard S, Shoorei H, Taheri M. miRNA profile in ovarian cancer. Exp Mol Pathol. 2020;113:104381.

11. Zhang Z, Zhang L, Wang B, Wei R, Wang Y, Wan J, Zhang C, Zhao L, Zhu $X$, Zhang $Y$, et al. MiR-337-3p suppresses proliferation of epithelial ovarian cancer by targeting PIK3CA and PIK3CB. Cancer Lett. 2020:469:54-67.

12. Pan C, Stevic I, Müller V, Ni Q, Oliveira-Ferrer L, Pantel K, Schwarzenbach H. Exosomal microRNAs as tumor markers in epithelial ovarian cancer. Mol Oncol. 2018;12(11):1935-48.

13. Kwon Y, Kim M, Kim Y, Jung HS, Jeoung D. Exosomal microRNAs as mediators of cellular interactions between cancer cells and macrophages. Front Immunol. 2020;11:1167.

14. Cheng $H$, Wang $Z$, Fu L, Xu T. Macrophage polarization in the development and progression of ovarian cancers: an overview. Front Oncol. 2019;9:421.

15. Travers M, Brown SM, Dunworth M. DFMO and 5-azacytidine increase M1 macrophages in the tumor microenvironment of murine ovarian cancer. Cancer Res. 2019;79(13):3445-3454.

16. Baig MS, Roy A, Rajpoot S, Liu D, Savai R, Banerjee S, Kawada M, Faisal SM, Saluja R, Saqib U, et al. Tumor-derived exosomes in the regulation of macrophage polarization. Inflamm Res. 2020;69(5):435-51.

17. An Y, Yang Q. MiR-21 modulates the polarization of macrophages and increases the effects of $\mathrm{M} 2$ macrophages on promoting the chemoresistance of ovarian cancer. Life Sci. 2020;242:117162.

18. Kalluri R. The biology and function of exosomes in cancer. J Clin Investig. 2016;126(4):1208-15

19. Wang J, Yao Y, Wu J, Li G. Identification and analysis of exosomes secreted from macrophages extracted by different methods. Int J Clin Exp Pathol. 2015;8(6):6135-42

20. Greening DW, Xu R, Ji H, Tauro BJ, Simpson RJ. A protocol for exosome isolation and characterization: evaluation of ultracentrifugation, densitygradient separation, and immunoaffinity capture methods. Methods Mo Biol. 2015:1295:179-209.

21. He C, Zheng S, Luo Y, Wang B. Exosome theranostics: biology and translational medicine. Theranostics. 2018;8(1):237-55.

22. Nakamura $K$, Sawada $K$, Kinose $Y$, Yoshimura A, Toda A, Nakatsuka E, Hashimoto K, Mabuchi S, Morishige KI, Kurachi H, et al. Exosomes promote ovarian cancer cell invasion through transfer of CD44 to peritoneal mesothelial cells. Mol Cancer Res. 2017;15(1):78-92.

23. Yoshimura A, Sawada K, Nakamura K, Kinose Y, Nakatsuka E, Kobayashi M, Miyamoto M, Ishida K, Matsumoto Y, Kodama M, et al. Exosomal miR-99a-5p is elevated in sera of ovarian cancer patients and promotes cancer cell invasion by increasing fibronectin and vitronectin expression in neighboring peritoneal mesothelial cells. BMC Cancer. 2018;18(1):1065.

24. Meng X, Müller V, Milde-Langosch K, Trillsch F, Pantel K, Schwarzenbach H. Circulating cell-free miR-373, miR-200a, miR-200b and miR-200c in patients with epithelial ovarian cancer. Adv Exp Med Biol. 2016;924:3-8.

25. Zuberi M, Mir R, Das J, Ahmad I, Javid J, Yadav P, Masroor M, Ahmad S, Ray PC, Saxena A. Expression of serum miR-200a, miR-200b, and miR200c as candidate biomarkers in epithelial ovarian cancer and their association with clinicopathological features. Clin Transl Oncol. 2015; 17(10):779-87.

26. Wang $A Q$, Lv M, Xu YH, Xie PM, Dong YY. MiR-200b-5p inhibits proliferation of ovarian cancer cells by targeting ATAD2 and regulating PI3K/AKT signaling pathway. Eur Rev Med Pharmacol Sci. 2020;24(19):9860-8. 
27. Funes SC, Rios M, Escobar-Vera J, Kalergis AM. Implications of macrophage polarization in autoimmunity. Immunology. 2018;154(2):186-195.

28. Lin L, Lin H, Wang L, Wang B, Hao X, Shi Y. miR-130a regulates macrophage polarization and is associated with non-small cell lung cancer. Oncol Rep. 2015;34(6):3088-96.

29. Carroll MJ, Kapur A, Felder M, Patankar MS, Kreeger PK. M2 macrophages induce ovarian cancer cell proliferation via a heparin binding epidermal growth factor/matrix metalloproteinase 9 intercellular feedback loop. Oncotarget. 2016;7(52):86608-20.

30. Jiang B, Zhu SJ, Xiao SS, Xue M. MiR-217 inhibits M2-like macrophage polarization by suppressing secretion of interleukin-6 in ovarian cancer. Inflammation. 2019;42(5):1517-29.

31. Hsu LS, Huang RH, Lai HW, Hsu HT, Sung WW, Hsieh MJ, Wu CY, Lin YM, Chen MK, Lo YS, et al. KLF6 inhibited oral cancer migration and invasion via downregulation of mesenchymal markers and inhibition of MMP-9 activities. Int J Med Sci. 2017;14(6):530-5.

32. Bi J, Zeng X, Zhao L, Wei Q, Yu L, Wang X, Yu Z, Cao Y, Shan F, Wei M. miR181a induces macrophage polarized to M2 phenotype and promotes M2 macrophage-mediated tumor cell metastasis by targeting KLF6 and C/EBPa. Mol Ther Nucleic Acids. 2016;5(9):e368.

33. Zhang S, Zhang JY, Lu L, Wang CH, Wang LH. MiR-630 promotes epithelial ovarian cancer proliferation and invasion via targeting KLF6. Eur Rev Med Pharmacol Sci. 2017;21(20):4542-4547.

\section{Publisher's Note}

Springer Nature remains neutral with regard to jurisdictional claims in published maps and institutional affiliations.

Ready to submit your research? Choose BMC and benefit from:

- fast, convenient online submission

- thorough peer review by experienced researchers in your field

- rapid publication on acceptance

- support for research data, including large and complex data types

- gold Open Access which fosters wider collaboration and increased citations

- maximum visibility for your research: over $100 \mathrm{M}$ website views per year

At BMC, research is always in progress.

Learn more biomedcentral.com/submissions 